\title{
Prevalence and clinical features of the ectopic ACTH syndrome in patients with gastroenteropancreatic and thoracic neuroendocrine tumors
}

\section{K Kamp ${ }^{1}$, R A Alwani ${ }^{1}$, E Korpershoek ${ }^{2}$, G J H Franssen ${ }^{3}$, W W de Herder ${ }^{1}$ and $\mathbf{R}$ A Feelders ${ }^{1}$}

${ }^{1}$ Sector of Endocrinology, Department of Internal Medicine, ${ }^{2}$ Department of Pathology and ${ }^{3}$ Department of Surgery, ENETS Center of Excellence, Erasmus Medical Center, 's-Gravendijkwal 230, 3015 CE Rotterdam, The Netherlands
Correspondence should be addressed to K Kamp

Email

k.kamp@erasmusmc.nl

\begin{abstract}
Objective: Several series report on the relative contribution of ectopic ACTH syndrome (EAS) in the spectrum of Cushing's syndrome. However, prevalence of EAS in patients with thoracic or gastroenteropancreatic neuroendocrine tumors (GEP-NETs) is currently unknown.

Design: We assessed, in a tertiary referral center, the prevalence of EAS in a large cohort of thoracic and GEP-NET patients including clinical, biochemical, and radiological features; management; and treatment outcome.

Methods: In total, 918 patients with thoracic or GEP-NETs were studied (1993-2012). Multiple endocrine neoplasia type 1 and small cell lung carcinoma patients were excluded. Differentiation between synchronous, metachronous, and cyclic occurrence of EAS was made.

Results: Out of the 918 patients with thoracic and GEP-NETs (469 males and 449 females; median age 58.7 years (range: 17.3-87.3)), 29 patients (3.2\%) had EAS (ten males and 19 females; median age 48.1 years (range: 24.7-77.9)). EAS occurred synchronously in 23 patients (79\%), metachronously in four patients (14\%), and cyclical in two patients (7\%) respectively. NETs causing EAS included lung/bronchus $(n=9)$, pancreatic $(n=9)$, and thymic $(n=4)$. In four patients, the cause of EAS was unknown $(n=4)$. Median overall survival (OS) of non-EAS thoracic and GEP-NET patients was 61.2 months (range: 0.6-249.4). Median OS of EAS patients was 41.4 months (range: 2.2-250.9). After comparison, only the first 5-year survival was significantly shorter $(P=0.013)$ in EAS patients.

Conclusion: Prevalence of EAS in this large cohort of patients with thoracic and GEP-NETs was 3.2\%. EAS was mostly caused by thoracic and pancreatic NETs. First 5-year survival of EAS patients was shorter compared with non-EAS patients.
\end{abstract}

\section{Introduction}

Cushing's syndrome (CS) is a rare and severe endocrine disorder characterized by a variety of typical signs and symptoms that occur due to chronic overproduction of cortisol. The estimated prevalence is two to three cases per million population per year (1).

Chronic hypercortisolism is associated with multiple systemic complications resulting in significant morbidity (c) 2016 European Society of Endocrinology Printed in Great Britain that severely impairs quality of life and an increased mortality when cortisol levels are not or suboptimally controlled (1).

CS is divided into adrenocorticotropin (ACTH)dependent CS, and ACTH-independent CS. In $\sim 80 \%$ of the cases, CS is ACTH-dependent, caused by either an ACTH-secreting pituitary adenoma (Cushing's disease) or,

Published by Bioscientifica Ltd. 
less often, by a non-pituitary ectopic ACTH-secreting tumor. ACTH-independent CS is caused by benign or malignant adrenal tumors or bilateral adrenal hyperplasia (1).

The ectopic ACTH syndrome (EAS) represents $20 \%$ of ACTH-dependent CS and about $10 \%$ of all types of CS $(1,2,3,4)$. EAS is associated with a variety of malignancies, predominantly of (neuro-)endocrine origin (bronchial, thymic, or pancreatic neuroendocrine tumors (NETs)). Other tumors associated with EAS are small cell lung carcinoma (SCLC), pheochromocytoma, medullary thyroid carcinoma (MTC), and prostate carcinoma $(2,5,6,7,8)$.

NETs originate from cells of the diffuse endocrine system and form a heterogeneous group of relatively rare neoplasms with various clinical manifestations and a distinct biological behavior (9). Recent epidemiologic studies show that the age-adjusted incidence of all gastroenteropancreatic (GEP)-NETs is 3.65 , for pancreatic NETs 0.43 , for bronchial NETs between 0.2 and 2.0, and for thymic NETs, this is $0.4 / 100000$ population per year $(10,11)$. Therefore, ectopic ACTH-secreting thoracic and GEP-NETs are extremely rare. Several large series report on the relative contribution of EAS in the spectrum of CS $(2,7,12)$. However, information on the incidence and prevalence of EAS in the setting of patients diagnosed with thoracic or GEP-NETs is currently unknown.

Therefore, the aim of this study was to assess the prevalence of EAS in a large cohort of patients with thoracic and GEP-NETs in a single tertiary academic referral center and to compare the prognosis in patients with and without EAS. Furthermore, clinical, biochemical, and radiological features; management; and treatment outcome of this patient cohort with EAS was evaluated.

\section{Patients and methods}

\section{Patients}

Patients with thoracic and GEP-NETs were identified from the Erasmus MC NET database. Patients diagnosed with the multiple endocrine neoplasia type 1 (MEN1) syndrome were excluded. Thymic NETs are known to be associated with MEN1, and MEN1-related ACTH-secreting NETs can harbor germline menin or other somatic mutations (1).

Also patients with SCLC, prostate carcinoma, and MTC were excluded from the study. The medical records of 918 (non-MEN1) patients with thoracic and GEP-NETs, evaluated between 1993 and 2012 in the Erasmus MC, Rotterdam, The Netherlands, were reviewed. All thoracic and GEP-NET patients treated in the Erasmus MC,
Rotterdam (as described in the present manuscript) gave written informed consent before inclusion in the studies, which were approved by the Medical Ethics Committee of the Erasmus MC, Rotterdam.

Analysis of clinical and pathological data, laboratory parameters, and imaging findings was performed, and information on medical and surgical treatments was collected.

\section{Diagnosis of thoracic NET and GEP-NET}

Diagnosis of thoracic NET and GEP-NET was made on the basis of serological markers (chromogranin A and neuronspecific enolase), pathologic elevations of circulating, hypersecreted neuroendocrine hormones, or peptides (13), imaging according to international protocols and standards $(14,15)$ in combination with histological confirmation according to current guidelines $(16,17)$.

\section{Diagnosis of EAS}

The diagnosis of CS was based on a review of the patient's medical history, clinical features, and laboratory tests. The use of exogenous glucocorticoids was excluded before biochemical tests were conducted. Hypercortisolism was biochemically established by elevated excretion of 24-h urinary free cortisol (UFC) levels on at least two occasions, insufficient suppression of serum cortisol after $1 \mathrm{mg}$ overnight dexamethasone (cutoff plasma cortisol (0800 h): $50 \mathrm{nmol} / \mathrm{l})$, loss of physiological cortisol diurnal rhythm (CDR) with assessment of midnight plasma and/or salivary cortisol levels, and non-suppressed plasma ACTH levels in the presence of normal or elevated plasma cortisol levels.

After the diagnosis of ACTH-dependent CS, the following tests were used to differentiate between pituitary and ectopic ACTH production: first, radiological imaging of the pituitary by magnetic resonance imaging (MRI); secondly, when no adenoma was present on radiological imaging or if the size of the adenoma was $<6 \mathrm{~mm}$, bilateral inferior petrosal sinus sampling (BIPSS) was performed as the gold standard differentiation test. The i.v. $7 \mathrm{mg}$ high-dose dexamethasone suppression test (HDDST) and the corticotropin-releasing hormone (CRH) stimulation test were not routinely used anymore from 2000 and onwards because of availability and better diagnostic performance of BIPSS (18).

\section{Definition of synchronous, metachronous, and cyclic EAS}

Further differentiation was made between synchronous (diagnosed within 3 months before or after thoracic or 
GEP-NET diagnosis), metachronous (diagnosed >3 months after thoracic or GEP-NET diagnosis), and cyclical EAS (repeated episodes of hypercortisolism intermediated by phases of normal cortisol secretion) based on the time between the diagnosis of EAS and the diagnosis of the thoracic or GEP-NET.

\section{Assays and investigations}

Serum cortisol (reference range: $200-700 \mathrm{nmol} / \mathrm{l}$ ), 24-h UFC excretion (reference range: $0-850 \mathrm{nmol} / 24 \mathrm{~h}$ ), and plasma ACTH (reference range: 0-11 pmol/l) were measured using chemiluminescence-based immunoassays (Immulite 2000, Siemens, Los Angeles, CA, USA; inter- and intra-assay coefficients of variation respectively below 15 and 7\% for cortisol and below 6.5 and 5.5\% for ACTH). Urinary cortisol was measured without prior solvent extraction. Although this may be a less accurate method, the upper limit of normal of this in-house assay was carefully determined according to cortisol secretion rate in healthy controls (19). Salivary cortisol was measured using a commercial kit (Salivary Cortisol ELISA SLV-2930, DRG Instruments $\mathrm{GmbH}$, Marburg, Germany).

\section{Statistical analysis}

Thoracic and GEP-NET patients with EAS were compared to those without EAS, regarding various clinicopathological parameters. Comparisons between the two groups were performed with unpaired $t$-test for numerical data and $\chi^{2}$ test for categorical data. The Fisher's exact test was used when the $\chi^{2}$ test had one or more cells with an expected frequency of five or less. The level of statistical significance was set to 0.05. Overall survival (OS) and 5-year survival were analyzed using Kaplan-Meier methods. Log-rank testing was used to determine whether there was a statistically significant difference between the mortality in thoracic and GEP-NET patients with and without EAS. Calculations were performed using Statistical Package for Social Sciences (SPSS) Software V.21.0.

\section{Results}

\section{Patients}

From 1993 to 2012, a total of 953 NET patients were treated in our department. After exclusion of 24 MEN1, four SCLCs, two prostate carcinoma, one MTC, and four other non-proven NET patients, 918 consecutive (non-MEN1) patients - 469 men and 449 women (female-to-male ratio, 1:1) - with thoracic and GEP-NETs were studied.

The median age of the patients at the time of the thoracic and GEP-NET diagnosis was 58.7 years (range: 17.3-87.3). The median follow-up of the study population was 61.2 months (range: 0.6-250.0). The majority of these tumors originated from the small intestine (267 patients $-29.1 \%$ ), followed in order of frequency by the non-functioning pancreas NETs (221 - 24.1\%), lung/ bronchus (51 - 5.6\%), large intestine ( $47-5.1 \%)$, ileocecal (43 - 4.7\%), and insulinoma (41 - 4.5\%). In 131 (14.3\%) patients, the primary tumor remained unknown until the completion of the study. The great majority of patients $(81.8 \%)$ were diagnosed with ENETS stage IV disease. Information on tumor grading was available in 523 patients: 240 (26.1\%) were G1, 240 (26.1\%) were G2, and 43 (4.7\%) were G3 (Table 1).

Evaluation of the medical records of these 918 thoracic and GEP-NETs patients revealed EAS in 29 patients - 19 women and ten men (female-to-male ratio, 1.9:1), corresponding with a prevalence of $3.2 \%$.

Patients were divided into two groups: patients with and without the EAS with respect to the patient's sex, age at diagnosis, localization of the primary thoracic or GEP-NET, localization of the metastasis, tumor grade, and ENETS stage. Highly significant differences were seen for the following parameters: a higher median age at diagnosis of the primary NET in the non-EAS group (58.9 years vs 48.1 years, $P<0.001$ ), a higher prevalence of thoracic (lung/bronchus and thymic) NETs in the EAS group (31\% vs $4.7 \%, P<0.001$ ), a higher prevalence of small intestine NETs in the non-EAS group (30\% vs $0 \%, P<0.001)$, a higher prevalence of liver metastasis in the non-EAS group (78.7\% vs $55.2 \%, P$ 0.003), and a higher prevalence of lung metastasis in the EAS group (27.6\% vs 7.8\%, $P$ 0.002); grade 2 tumors were more frequently seen in patients in the EAS group $(48.3 \%$ vs 25.4\%, $P$ 0.006), and stage IIIB was more frequently present in the EAS group $(24.1 \%$ vs $9.3 \%, P 0.018)$, whereas stage IV was more frequently seen in the nonEAS group (82.5\% vs 62.1\%, P 0.005) (Table 1).

\section{Clinical presentation and complications of EAS}

The majority of patients presented with the classic clinical signs and symptoms of CS were caused by known ectopic ACTH secretion: muscle weakness (79\%), hypokalemia (72\%), body weight changes (69\%), truncal obesity (66\%), full moon face (66\%), hypertension (59\%), and diabetes (59\%) (Table 2). 
Table 1 Clinicopathological characteristics of 918 patients with thoracic and GEP-NETs evaluated for the presence of EAS. Group analysis has been conducted between thoracic and GEP-NET patients with and without the EAS.

\begin{tabular}{|c|c|c|c|c|c|c|c|}
\hline \multirow{2}{*}{$\begin{array}{l}\text { Clinicopathological } \\
\text { characteristics }\end{array}$} & \multicolumn{2}{|c|}{ All patients $(n=918)$} & \multicolumn{2}{|c|}{ Non-Cushing NET $(n=889)$} & \multicolumn{2}{|c|}{$\begin{array}{c}\text { Ectopic ACTH } \\
\text { Cushing NET }(n=29)\end{array}$} & \multirow[b]{2}{*}{$P$ value } \\
\hline & $n$ & $\%$ & $n$ & $\%$ & $n$ & $\%$ & \\
\hline \multicolumn{8}{|l|}{ Gender } \\
\hline Male & 469 & 51.1 & 459 & 51.6 & 10 & 34.5 & 0.069 \\
\hline Female & 449 & 48.9 & 430 & 48.4 & 19 & 65.5 & \\
\hline Age at diagnosis NET (years) & \multicolumn{2}{|c|}{$58.7(17.3-87.3)$} & \multicolumn{2}{|c|}{$58.9(17.3-87.3)$} & \multicolumn{2}{|c|}{$48.1(24.7-77.9)$} & $<0.001$ \\
\hline$<50$ & 205 & 22.3 & 189 & 21.3 & 16 & 55.2 & $<0.001$ \\
\hline $50-69$ & 555 & 60.5 & 543 & 61.1 & 12 & 41.4 & 0.033 \\
\hline$>70$ & 158 & 17.2 & 157 & 17.7 & 1 & 3.4 & 0.045 \\
\hline \multicolumn{8}{|l|}{ Primary localization } \\
\hline Lung/bronchus & 51 & 5.6 & 42 & 4.7 & 9 & 31 & $<0.001$ \\
\hline Thymus & 6 & 0.7 & 2 & 0.2 & 4 & 13.8 & $<0.001$ \\
\hline Stomach & 18 & 2 & 17 & 1.9 & 1 & 3.4 & 0.442 \\
\hline Small intestine & 267 & 29.1 & 267 & 30 & 0 & 0 & $<0.001$ \\
\hline Appendix & 16 & 1.7 & 15 & 1.7 & 1 & 3.4 & 0.404 \\
\hline Ileocecal & 43 & 4.7 & 43 & 4.8 & 0 & 0 & 0.393 \\
\hline Large intestine & 47 & 5.1 & 47 & 5.3 & 0 & 0 & 0.394 \\
\hline Rectum & 32 & 3.5 & 32 & 3.6 & 0 & 0 & 0.62 \\
\hline Other ${ }^{a}$ & 12 & 1.3 & 12 & 1.3 & 0 & 0 & 1.000 \\
\hline Cancer of unknown primary & 131 & 14.3 & 127 & 14.3 & 4 & 13.8 & 1.000 \\
\hline \multicolumn{8}{|l|}{ Pancreas } \\
\hline Non-functioning & 221 & 24.1 & 212 & 23.8 & 9 & 31 & 0.373 \\
\hline Insulinoma & 41 & 4.5 & 41 & 4.6 & 0 & 0 & 0.636 \\
\hline Glucagonoma & 6 & 0.7 & 6 & 0.7 & 0 & 0 & 1.000 \\
\hline Gastrinoma & 16 & 1.7 & 15 & 1.7 & 1 & 3.4 & 0.404 \\
\hline VIPoma & 10 & 1.1 & 10 & 1.1 & 0 & 0 & 1.000 \\
\hline Somatostatinoma & 1 & 0.1 & 1 & 0.1 & 0 & 0 & 1.000 \\
\hline \multicolumn{8}{|l|}{ Metastasis localization } \\
\hline Lymph node & 648 & 70.6 & 631 & 71 & 17 & 58.6 & 0.151 \\
\hline Liver & 716 & 78 & 700 & 78.7 & 16 & 55.2 & 0.003 \\
\hline Bone & 214 & 23.3 & 207 & 23.3 & 7 & 24.1 & 0.915 \\
\hline Lung & 77 & 8.4 & 69 & 7.8 & 8 & 27.6 & 0.002 \\
\hline Other ${ }^{\mathrm{b}}$ & 127 & 13.8 & 123 & 13.8 & 4 & 13.8 & 1.000 \\
\hline \multicolumn{8}{|l|}{ Tumor grade } \\
\hline G1 & 240 & 26.1 & 236 & 26.5 & 4 & 13.8 & 0.124 \\
\hline $\mathrm{G} 2$ & 240 & 26.1 & 226 & 25.4 & 14 & 48.3 & 0.006 \\
\hline G3 & 43 & 4.7 & 40 & 4.5 & 3 & 10.3 & 0.15 \\
\hline Unknown & 395 & 43 & 387 & 43.5 & 8 & 27.6 & 0.088 \\
\hline \multicolumn{8}{|l|}{ ENETS stage } \\
\hline I-IIIa & 77 & 8.4 & 73 & 8.2 & 4 & 13.8 & 0.296 \\
\hline IIIb & 90 & 9.8 & 83 & 9.3 & 7 & 24.1 & 0.018 \\
\hline IV & 751 & 81.8 & 733 & 82.5 & 18 & 62.1 & 0.005 \\
\hline
\end{tabular}

${ }^{a}$ Other primary tumors included: oesophagus, kidney, and ovary NETs.

${ }^{b}$ Other metastasis included: adrenal, heart, brain, spleen, breast, skin, thyroid, testis, eye, and uterus.

Complications due to severe hypercortisolism included uncontrolled diabetes (59\%) and severe or opportunistic infections (41\%) involving the skin $(n=2)$, urogenital tract $(n=3)$, respiratory tract $(n=5)$, sepsis $(n=3)$, herpes zoster $(n=1)$, and candida $(n=1)$. Three patients had multiple recurrent infections. Other complications were severe hypertension (17\%), pulmonary embolism $(n=3)$ or thrombosis $(n=1)(14 \%)$, and psychosis (14\%) (Table 2).

\section{Diagnosis of EAS}

All patients but one (due to failure in urine collection) had elevated excretion of 24-h UFC levels above the normal reference range on at least two occasions: median baseline UFC - $5872 \mathrm{nmol} / 24 \mathrm{~h}$ (range: 1028-89 $392 \mathrm{nmol} / 24 \mathrm{~h}$ ) and median highest UFC - $14405 \mathrm{nmol} / 24 \mathrm{~h}$ (range: 1127-30 $149 \mathrm{nmol} / 24 \mathrm{~h}$ ). All patients had insufficient suppression of serum cortisol after $1 \mathrm{mg}$ overnight 
Table 2 Clinical symptoms and signs at presentation including complications in patients with EAS $(n=29)$.

\begin{tabular}{|c|c|c|}
\hline Clinical presentation and complications & $n$ & $\%$ \\
\hline \multicolumn{3}{|l|}{ Clinical symptoms and signs } \\
\hline Muscle weakness & 23 & 79 \\
\hline Hypokalemia & 21 & 72 \\
\hline Body weight & 20 & 69 \\
\hline Increase & 17 & 59 \\
\hline Decrease & 3 & 10 \\
\hline Truncal obesity & 19 & 66 \\
\hline Full moon face & 19 & 66 \\
\hline Hypertension & 17 & 59 \\
\hline Diabetes & 17 & 59 \\
\hline Edema & 16 & 55 \\
\hline Bruising & 15 & 52 \\
\hline Hirsutism & 14 & 48 \\
\hline Buffalo hump & 13 & 45 \\
\hline Psychiatric disorders & 11 & 38 \\
\hline Osteopenia or osteoporosis & 9 & 31 \\
\hline Acne & 7 & 24 \\
\hline Hyperpigmentation & 7 & 24 \\
\hline Insomnia & 6 & 21 \\
\hline Impaired cognition or memory & 5 & 17 \\
\hline Violaceous striae & 4 & 14 \\
\hline Menstrual irregularities or amenorrhea & 2 & 7 \\
\hline Libido & 2 & 7 \\
\hline Fractures & 2 & 7 \\
\hline \multicolumn{3}{|l|}{ Complications } \\
\hline Uncontrolled diabetes & 17 & 59 \\
\hline Severe or opportunistic infections & 12 & 41 \\
\hline Severe hypertension & 5 & 17 \\
\hline Thrombosis or pulmonary embolism & 4 & 14 \\
\hline Psychosis & 4 & 14 \\
\hline
\end{tabular}

dexamethasone (cutoff $50 \mathrm{nmol} / \mathrm{l}$ ), loss of physiological CDR, and non-suppressed plasma ACTH levels (median $37.9 \mathrm{pmol} / \mathrm{l}$, range: $1.8-169.0 \mathrm{pmol} / \mathrm{l})$ in the presence of normal or elevated serum cortisol levels (median $1091 \mathrm{nmol} / \mathrm{l}$, range: $324-3707 \mathrm{nmol} / \mathrm{l})$.

For further differentiation between pituitary and ectopic ACTH production, 16 of 29 (55\%) patients underwent MRI of the pituitary gland, but in no case did this show any clear evidence of an adenoma at presentation or follow-up. Overall, 20 patients underwent a HDDST, and 19 of 20 (95\%) showed no serum cortisol suppression after HDSST. However, the only patient who showed cortisol suppression after HDSST (42.5\% suppression of the baseline value) was diagnosed with a histologically proven EAS (lung NET). Data on the CRH stimulation test were available for 14 patients: 13 exhibited no cortisol or ACTH response after CRH administration; however, the same patient with a lung NET that showed cortisol suppression after HDSST also demonstrated after human CRH administration a 14 and $23 \%$ cortisol rise at 30 and 45 min respectively.
Eight patients underwent BIPSS, none of them showed a central-to-peripheral ACTH gradient.

In most patients, EAS was identified synchronously within 3 months before or after the thoracic or GEP-NET diagnosis $(n=23)$. Metachronous EAS was only seen in few patients $(n=4)$. In two patients, cortisol levels fluctuated markedly during follow-up (repeated episodes of hypercortisolism intermediated by phases of normal cortisol secretion), indicating cyclical EAS.

These two patients with cyclical EAS were diagnosed with a histologically proven thymic NET and pancreatic neuroendocrine tumor (PanNet) respectively.

\section{Radiological investigation}

A variety of imaging modalities was used over time to localize ACTH-producing thoracic or GEP-NET, as different imaging techniques became available. In total, 27 EAS patients underwent chest radiography, which revealed the presence of the primary thoracic tumor in six patients and metastases in only two out of eight patients with lung metastases. In total, 25 EAS patients underwent computed tomography (CT) imaging of the chest, which identified 13 with primary thoracic NETs, and 28 EAS patients had CT imaging of the abdomen, which identified eight of 12 GEP-NETs. Five EAS patients, who underwent CT imaging of the abdomen, additionally underwent MRI of the abdomen, and this revealed one with more primary pancreatic NET and other patient with metastases of the liver.

Octreotide scanning ( ${ }^{111} \mathrm{In}$-pentetreotide scintigraphy) was performed in all 29 EAS patients, 20 had positive octreotide scintigraphy. In total, four of these 20 patients had an octreoscan that did not reveal the primary tumor but did reveal the presence of metastases (lymph nodes and liver). Two patients had an ${ }^{123}$ I-MIBG scan that did not reveal the primary in either but did reveal the presence of metastases (liver) in one patient. Positron emission tomography (PET), a relatively new, non-invasive technique, was performed in six EAS patients. One of these six patients, with a G3 lung NET, underwent $\left[{ }^{18} \mathrm{~F}\right]$-fluorodeoxyglucose PET which provided just limited information on lymphnode metastases. Additional ${ }^{68} \mathrm{Ga}$-DOTA-TOC imaging in this patient revealed metastases of liver and bones. ${ }^{18} \mathrm{~F}$-DOPA scan performed in three patients revealed the presence of already known liver metastases in one patient. In another patient, both ${ }^{18} \mathrm{~F}$-DOPA and ${ }^{11} \mathrm{C}-5$-HTP-PET scans were performed and were negative.

${ }^{11} \mathrm{C}-5$-HTP-PET imaging was able to identify the primary thymic NET in one of our patients. 


\section{Management of EAS}

Medical treatment - A total of 23 patients with EAS received medical treatment which consisted of the glucocorticoid receptor antagonist mifepristone, adrenolytic agents such as ketoconazole and etomidate, or ACTH inhibitory agents like somatostatin analogs (SSAs). Mifepristone, ketoconazole, and SSAs were used alone in five, three, and five patients respectively. Other patients received up to three drugs simultaneously or sequentially. Medication was discontinued or changed because of side effects $(n=4)$ or inadequate inhibition $(n=12)$. All patients $(n=18)$, except two, had persistent hypercortisolism under treatment with ketoconazole or deteriorated under mifepristone treatment, and 15 of these patients underwent bilateral adrenalectomy. Of the two patients in whom clinical symptoms improved under treatment with mifepristone, one underwent surgery of the primary tumor, whereas the other patient ultimately underwent a bilateral adrenalectomy.

Surgical treatment Curative resection or debulking of the primary tumor and its metastases was performed in 16 patients with EAS. Overall, seven patients had a curative resection and were cured of EAS; however, two of these patients previously underwent a bilateral adrenalectomy to control their hypercortisolism. In the other nine patients, surgery was not curative, and the EAS was controlled by bilateral adrenalectomy. In total, 22 out of 29 patients underwent a bilateral adrenalectomy to control the hypercortisolism caused by EAS.

Adjunctive therapy of the thoracic and GEP-NET Seven patients were treated with peptide receptor radiotherapy, six patients (three PanNet, two unknown primary NETs, and one thymic NET) were treated with a median dose of ${ }^{177} \mathrm{Lu}$-octreotate of $29.0 \mathrm{GBq}$ (range: 14.8-44.9 GBq) divided over two to six therapy cycles, and one patient with an appendix NET was treated with a total dose of ${ }^{111}$ In-pentetreotide of $58.6 \mathrm{GBq}$ divided over eight therapy cycles. In two patients, ${ }^{177} \mathrm{Lu}$-octreotate was used as an adjuvant treatment to surgery, which resulted in two complete responses (23.4 and 34.4 months still ongoing at the end of this study). Other best responses of ${ }^{177} \mathrm{Lu}$-octreotate were two partial responses (25.1 and 57.1 months) and two stable disease responses (32.5 and 6 months). Information regarding the response and duration of response of the patient treated with ${ }^{111}$ In-pentetreotide therapy was not available.
Three patients with thymic NETs received external radiotherapy to the mediastinum. In three GEP-NET patients, radiotherapy was directed to the bone metastases for pain control. Other adjunctive treatments were cytotoxic chemotherapy (10.3\%) and everolimus (6.9\%).

\section{Pathological findings}

Of the 29 EAS thoracic and GEP-NET patients, 16 patients had a histopathological-established diagnosis at Erasmus MC, Rotterdam, with the exception of seven patients who had a histopathological diagnosis before referral. Our pathology department reviewed all tumor tissues, obtained from the referring hospitals, in order to confirm the diagnosis. Furthermore, ACTH immunohistochemistry was positive in 13 out of 18 patients.

\section{Prognosis and survival}

At the last time point of follow-up, 15 of 29 (52\%) EAS NET patients and 547 of 889 (62\%) non-EAS thoracic and GEP-NET patients were still alive. In the EAS NET group, patients died of complications due to progression of the tumor itself $(n=8)$ or as a consequence of previous excessive cortisol secretion (opportunistic infections $(n=2)$, cardiac failure $(n=2)$, and pulmonary embolism $(n=1))$; in one patient, the cause of death remained unknown. From this total of 14 deaths in the EAS NET group, two patients did not undergo bilateral adrenalectomy (one patient was in poor clinical condition and died within 2 months; the other patient refused and died after 12 months), nine patients had an early bilateral adrenalectomy (within 1 month after diagnosis of EAS), and three patients had a late bilateral adrenalectomy (after more than 4 months). Median survival of patients after early bilateral adrenalectomy was 6.2 months (range: 5.0-20.9 months), and median survival of patients after late bilateral adrenalectomy was 5.2 months (range: 2.2-20.9 months).

Median OS of the non-EAS patients was 61.2 months (range: 0.6-249.4), whereas median OS of the EAS patients was 41.4 months (range: 2.2-250.9). The OS in the patients with EAS was not significantly shorter $(P=0.151)$ than in the group with non-EAS patients when compared for the complete duration of follow-up. However, the first 5-year survival of the EAS patients was significantly shorter $(P=0.013)$ than in the group with non-EAS patients (Fig. 1) 


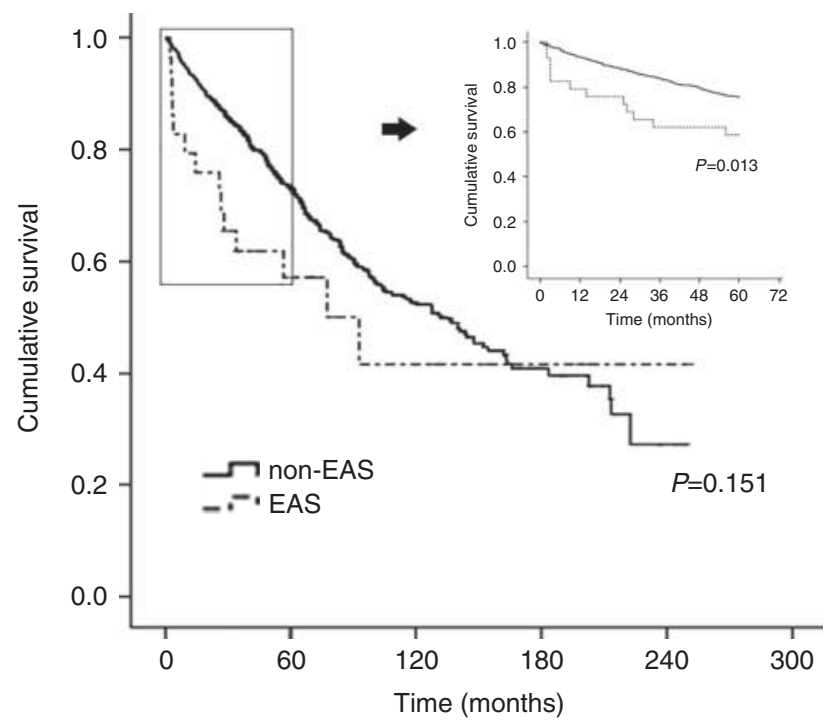

Figure 1

Overall survival and 5-year survival of EAS $(n=29)$ vs non-EAS thoracic and GEP-NET patients $(n=889)$.

Survival according to tumor grade (Fig. 2) and ENETS tumor stage (Fig. 3) compared in patients with and without EAS showed no significant differences.

\section{Discussion}

We have evaluated the prevalence of EAS in a large cohort of patients with thoracic and GEP-NETs, who were followed in a single, academic, and tertiary referral center. In our retrospective study, we found that, within a period of 20 years (1993-2012), 918 new patients with thoracic and GEP-NETs included 29 patients (3.2\%) with proven EAS.

In addition, we present the clinical, endocrine, and radiological features; management; and treatment outcome of our cohort of 29 thoracic and GEP-NET patients with EAS evaluated and treated within a single center.

In line with literature, thoracic NETs (lung/bronchus and thymic) were the most common tumors with ectopic ACTH production (44.8\%) in our study, followed by nonfunctioning PanNETs (31\%). Furthermore, EAS occurred only in one patient with an appendix NET in accordance with Ilias et al., which may explain why EAS is not a major concern in NETs originating from the midgut $(1,2,7)$.

Comparisons were made between thoracic and GEP-NET patients with and without EAS which showed highly significant differences regarding various clinicopathological parameters. The lower median age at diagnosis of EAS patients is presumably explained by an earlier onset of symptoms due to the EAS. This probably also clarifies why stage IIIB tumors, without distant metastasis, are more frequently seen in the EAS group. The differences in localization between the distant metastasis, lung for the EAS group and liver for the nonEAS group, are in line with the primary tumor site.

This series of 29 patients illustrates the broad clinical spectrum of EAS that can present with a variable clinical phenotype as can be inferred from the prevalence of signs and symptoms $(2,7,20)$.

In our cohort, all patients had disturbed first-line screenings tests for hypercortisolism. Although dynamic testing with CRH test and HDDST performed reasonably well, only BIPSS resulted in a sensitivity and specificity of $100 \%$ to differentiate between EAS and central ACTH overproduction.

CT or MRI of thorax and abdomen and octreoscan revealed the primary EAS lesion in most patients. Thoracic NETs were best detected by CT imaging of the chest

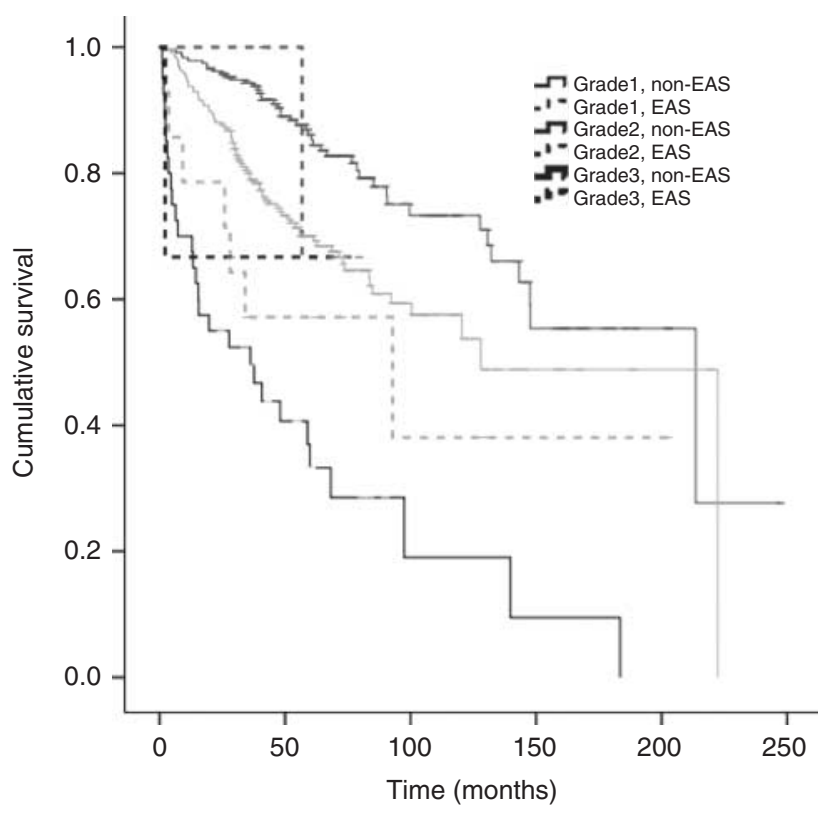

\section{Figure 2}

Survival curves by tumor grade (EAS vs non-EAS patients). Grade 1 EAS $(n=4)$ vs non-EAS $(n=236), P=0.664$; grade 2 EAS $(n=14)$ vs grade 2 non-EAS $(n=226), P=0.147$; and grade 3 EAS $(n=3)$ vs non-EAS $(n=40), P=0.502$. Grading: grade 1 non-EAS; grade 1 EAS; grade 2 non-EAS; grade 2 EAS; grade 3 non-EAS; grade 3 EAS. A full colour version of this figure is available at http://dx.doi.org/10.1530/EJE-15-0968. 


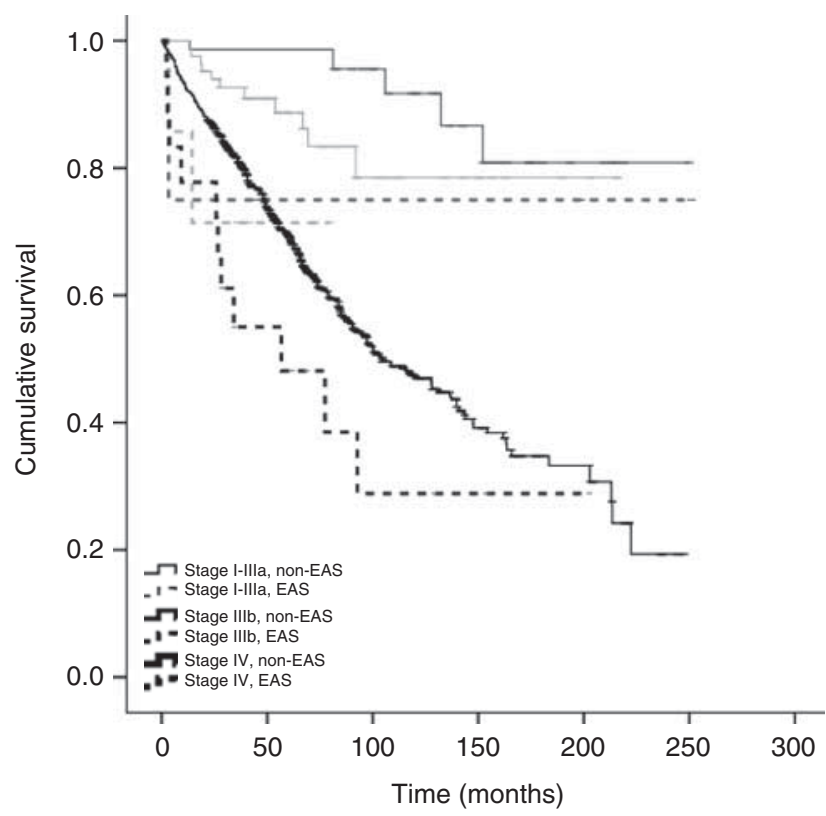

\section{Figure 3}

Survival curves by ENETS tumor stage (EAS vs non-EAS patients). Stage I-IIIA EAS $(n=4)$ vs non-EAS $(n=73), P=0.381$; stage IIIB EAS $(n=7)$ vs non-EAS $(n=83), P=0.182$; and stage IV EAS $(n=18)$ vs non-EAS $(n=733), P=0.064$. Staging: stage I-IIla non-EAS; stage I-IIla EAS; stage IIlb non-EAS; stage IIlb EAS; stage IV non-EAS; stage IV EAS. A full colour version of this figure is available at http://dx.doi.org/10.1530/EJE-15-0968.

(100\%) and GEP-NETs by CT or MRI of the abdomen (75\%). These results are in line with previously published studies that localized the primary tumor in $70-90 \%$ of the ectopic ACTH cases $(2,7)$. The octreoscan was positive in $69 \%$ of patients and showed no superiority over CT or MRI in detecting more lesions. NETs express somatostatin receptors (SSTs), in particular subtype 2, and can be identified with SSTs scintigraphy with ${ }^{111}$ In-pentetreotide octreoscan. In EAS patients, reported sensitivity of the diagnostic octreoscan varies between 25 and 80\%, and this broad range may in part be explained by differences in imaging technique protocols $(2,7,21,22)$.

Furthermore, we found five patients with negative ACTH immunohistochemistry in our series (lung $=2$ and PanNET =3). This is in line with Isidori et al., they state that, in all patients, diagnosis was further confirmed by positive ACTH immunoreactivity $(n=15)$ and/or complete/partial resolution of the hypercortisolemia after tumor removal/ debulking $(n=19)$. They also found negative ACTH immunohistochemistry in several (lung) NET patients (7). Another possible explanation could be bad fixation during immunohistochemistry in combination with the revision of relatively old tumor tissue samples.

Management of patients with ACTH-producing thoracic and GEP-NETs was aimed at control of cortisol excess, preferably also paralleled by tumor stabilization or reduction, resulting in prolongation of survival. Optimal primary treatment of EAS with surgical resection of the primary tumor and its metastasis was possible in $24 \%$ of our EAS patients. In second-line treatment to control cortisol excess, cortisol included bilateral adrenalectomy, medical therapy, and radiotherapy $(1,2,6,7,23,24)$. Ultimately, most of our patients underwent bilateral adrenalectomy to control hypercortisolism.

Two large historical case record studies evaluated survival of EAS in NETs and other tumors. In the study by Ilias et al. (2), a cumulative survival of $\sim 85 \%$ in pulmonary NET patients at 5 years was shown. In the study by Isidori et al. (7), cumulative survival of NET at 5 years without distant metastases was about $80 \%$ and with distant metastases (stage IV) $60 \%$. OS in our cohort of 29 EAS patients (62\% stage IV) was $65 \%$ at 5 years and for non-EAS patients (82.5\% stage IV) $75 \%$ at 5 years. We conclude that, in our cohort, OS of patients with EAS was not significantly shorter than OS of non-EAS patients when compared for the complete duration of follow-up. However, the first 5-year survival of EAS patients was significantly shorter than the first 5-year survival of nonEAS patients. This difference could be explained by the proportion of 'cured' patients in the EAS group, in which survival is probably no longer largely determined by the EAS or the thoracic or GEP-NET. Literature shows that the overall prognosis of EAS patients is mainly determined by the tumor grade and stage at the time of diagnosis (2). In our study, eight EAS patients died of complications due to progression of the tumor itself, whereas five patients died due to complications of excessive cortisol secretion. Chronic hypercortisolism can induce multisystem morbidities and serious complications (25). Cardiovascular risk factors (e.g. uncontrolled diabetes, severe hypertension, and obesity), immunosuppressive effects increasing the risk of opportunistic infections and sepsis, and venous thromboembolism occurred frequently in our patient cohort.

Although we report the prevalence of EAS in a very large cohort of patients with thoracic and GEP-NETs, our study has a minor limitation, since not all patients with thoracic and GEP-NETs were screened for hypercortisolism and EAS. Therefore, hypothetically patients with mild EAS might not have been diagnosed. However, EAS is usually symptomatic and associated with severe hypercortisolism. 
In this retrospective study, we conclude that, within a period of 20 years (1993-2012), the prevalence of EAS in a large cohort of patients with sporadic thoracic and GEP-NETs was 3.2\%. The first 5-year survival was shorter in patients with EAS compared with non-EAS patients. Our and other studies show that EAS in patients with thoracic and GEP-NETs is associated with serious morbidity and a high mortality risk. Therefore, aggressive treatment of hypercortisolism with (combination) medical therapy or rescue bilateral adrenalectomy is an essential part of patient management.

\section{Declaration of interest}

The authors declare that there is no conflict of interest that could be perceived as prejudicing the impartiality of the research reported.

\section{Funding}

This research did not receive any specific grant from any funding agency in the public, commercial or not-for-profit sector.

\section{Author contribution statement}

All authors contributed to conception and design. K Kamp contributed to collection and assembly of data. All authors contributed to data analysis and interpretation, manuscript writing, and final approval of manuscript.

\section{Acknowledgements}

We thank Martijn den Dekker and Wanda Geilvoet for their excellent contributions to this study.

\section{References}

1 Lacroix A, Feelders RA, Stratakis CA \& Nieman LK. Cushing's syndrome. Lancet $2015386913-927$.

2 Ilias I, Torpy DJ, Pacak K, Mullen N, Wesley RA \& Nieman LK. Cushing's syndrome due to ectopic corticotropin secretion: twenty years' experience at the National Institutes of Health. Journal of Clinical Endocrinology and Metabolism 200590 4955-4962. (doi:10.1210/jc. 2004-2527)

3 Newell-Price J, Bertagna X, Grossman AB \& Nieman LK. Cushing's syndrome. Lancet 2006367 1605-1617. (doi:10.1016/S01406736(06)68699-6)

4 Tritos NA, Biller BM \& Swearingen B. Management of Cushing disease. Nature Reviews. Endocrinology 20117 279-289. (doi:10.1038/nrendo. 2011.12)

5 Phan AT \& Yao JC. Neuroendocrine tumors: novel approaches in the age of targeted therapy. Oncology 200822 1617-1623 (discussion 1623-1624, 1629).

6 Biller BM, Grossman AB, Stewart PM, Melmed S, Bertagna X, Bertherat J, Buchfelder M, Colao A, Hermus AR, Hofland LJ et al. Treatment of adrenocorticotropin-dependent Cushing's syndrome: a consensus statement. Journal of Clinical Endocrinology and Metabolism 200893 2454-2462. (doi:10.1210/jc.2007-2734)
7 Isidori AM, Kaltsas GA, Pozza C, Frajese V, Newell-Price J, Reznek RH, Jenkins PJ, Monson JP, Grossman AB \& Besser GM. The ectopic adrenocorticotropin syndrome: clinical features, diagnosis, management, and long-term follow-up. Journal of Clinical Endocrinology and Metabolism 200691 371-377. (doi:10.1210/jc.2005-1542)

8 Alwani RA, Neggers SJ, van der Klift M, Baggen MG, van Leenders GJ, van Aken MO, van der Lely AJ, de Herder WW \& Feelders RA. Cushing's syndrome due to ectopic ACTH production by (neuroendocrine) prostate carcinoma. Pituitary 200912 280-283. (doi:10.1007/s11102-008-0100-z)

9 Modlin IM, Oberg K, Chung DC, Jensen RT, de Herder WW, Thakker RV, Caplin M, Delle Fave G, Kaltsas GA, Krenning EP et al. Gastroenteropancreatic neuroendocrine tumours. Lancet. Oncology 20089 61-72. (doi:10.1016/S1470-2045(07)70410-2)

10 Fraenkel M, Kim M, Faggiano A, de Herder WW, Valk GD \& Knowledge N. Incidence of gastroenteropancreatic neuroendocrine tumours: a systematic review of the literature. Endocrine-Related Cancer 201421 R153-R163. (doi:10.1530/ERC-13-0125)

11 Yao JC, Hassan M, Phan A, Dagohoy C, Leary C, Mares JE, Abdalla EK, Fleming JB, Vauthey JN, Rashid A et al. One hundred years after "carcinoid": epidemiology of and prognostic factors for neuroendocrine tumors in 35,825 cases in the United States. Journal of Clinical Oncology 200826 3063-3072. (doi:10.1200/JCO.2007.15.4377)

12 Aniszewski JP, Young WF Jr, Thompson GB, Grant CS \& van Heerden JA. Cushing syndrome due to ectopic adrenocorticotropic hormone secretion. World Journal of Surgery 200125 934-940. (doi:10.1007/s00268-001-0032-5)

13 O'Toole D, Grossman A, Gross D, Delle Fave G, Barkmanova J, O'Connor J, Pape UF, Plockinger U, Mallorca Consensus Conference participants \& European Neuroendocrine Tumor Society. ENETS Consensus Guidelines for the Standards of Care in Neuroendocrine Tumors: biochemical markers. Neuroendocrinology 200990 194-202. (doi:10.1159/000225948)

14 Kwekkeboom DJ, Krenning EP, Scheidhauer K, Lewington V, Lebtahi R, Grossman A, Vitek P, Sundin A, Plockinger U, Mallorca Consensus Conference participants \& European Neuroendocrine Tumor Society. ENETS Consensus Guidelines for the Standards of Care in Neuroendocrine Tumors: somatostatin receptor imaging with (111)In-pentetreotide. Neuroendocrinology 200990 184-189. (doi:10.1159/000225946)

15 Sundin A, Vullierme MP, Kaltsas G, Plockinger U, Mallorca Consensus Conference participants \& European Neuroendocrine Tumor Society. ENETS Consensus Guidelines for the Standards of Care in Neuroendocrine Tumors: radiological examinations. Neuroendocrinology 200990 167-183. (doi:10.1159/000184855)

16 Klimstra DS, Modlin IR, Coppola D, Lloyd RV \& Suster S. The pathologic classification of neuroendocrine tumors: a review of nomenclature, grading, and staging systems. Pancreas 201039 707-712. (doi:10.1097/MPA.0b013e3181ec124e)

17 Kloppel G, Couvelard A, Perren A, Komminoth P, McNicol AM, Nilsson O, Scarpa A, Scoazec JY, Wiedenmann B, Papotti M et al. ENETS Consensus Guidelines for the Standards of Care in Neuroendocrine Tumors: towards a standardized approach to the diagnosis of gastroenteropancreatic neuroendocrine tumors and their prognostic stratification. Neuroendocrinology 200990 162-166. (doi:10.1159/000182196)

18 van den Bogaert DP, de Herder WW, de Jong FH, Biemond P, van der Lely AJ \& Lamberts SW. The continuous 7-hour intravenous dexamethasone suppression test in the differential diagnosis of ACTH-dependent Cushing's syndrome. Clinical Endocrinology 199951 193-198. (doi:10.1046/j.1365-2265.1999.00759.x)

19 Croughs RJ, Docter R \& de Jong FH. Comparison of oral and intravenous dexamethasone suppression tests in the differential diagnosis of Cushing's syndrome. Acta Endocrinologica 197372 54-62.

20 Nieman LK, Biller BM, Findling JW, Newell-Price J, Savage MO, Stewart PM \& Montori VM. The diagnosis of Cushing's syndrome: an Endocrine Society Clinical Practice Guideline. Journal of Clinical Endocrinology and Metabolism 200893 1526-1540. (doi:10.1210/jc. 2008-0125 
21 de Herder WW, Krenning EP, Malchoff CD, Hofland LJ, Reubi JC, Kwekkeboom DJ, Oei HY, Pols HA, Bruining HA, Nobels FR et al. Somatostatin receptor scintigraphy: its value in tumor localization in patients with Cushing's syndrome caused by ectopic corticotropin or corticotropin-releasing hormone secretion. American Journal of Medicine 199496 305-312. (doi:10.1016/0002-9343(94)90059-0)

22 Tabarin A, Valli N, Chanson P, Bachelot Y, Rohmer V, Bex-Bachellerie V, Catargi B, Roger P \& Laurent F. Usefulness of somatostatin receptor scintigraphy in patients with occult ectopic adrenocorticotropin syndrome. Journal of Clinical Endocrinology and Metabolism 199984 1193-1202. (doi:10.1210/jcem.84.4.5583)
23 van der Pas R, de Herder WW, Hofland LJ \& Feelders RA.

New developments in the medical treatment of Cushing's syndrome. Endocrine-Related Cancer 201219 R205-R223. (doi:10.1530/ ERC-12-0191)

24 Reincke M, Ritzel K, Osswald A, Berr CM, Stalla GK, Hallfeldt K, Reisch N, Schopohl J \& Beuschlein F. A critical re-appraisal of bilateral adrenalectomy for ACTH-dependent Cushing's syndrome. European Journal of Endocrinology 2015173 M23-M32. (doi:10.1530/EJE-15-0265)

25 Feelders RA, Pulgar SJ, Kempel A \& Pereira AM. The burden of Cushing's disease: clinical and health-related quality of life aspects. European Journal of Endocrinology 2012167 311-326. (doi:10.1530/EJE-11-1095)

Received 29 September 2015

Revised version received 13 November 2015

Accepted 7 December 2015 